\title{
Ética del cuidado y cuidado de enfermería
}

\author{
Ethics of Care and Nursing Care
}

\section{Ética do cuidado e cuidado de enfermagem}

\author{
Kathia Yáñez Flores ${ }^{1}$, ORCID 0000-0003-1524-1133 \\ Edith Rivas Riveros ${ }^{2}$, ORCID 0000-0002-9832-4534 \\ Maggie Campillay Campillay ${ }^{3}$, ORCID 0000-0002-4054-1595 \\ ${ }^{12}$ Universidad de La Frontera. Chile \\ ${ }^{3}$ Universidad de Atacama. Chile
}

Recibido: 08/04/2020

Aceptado: 03/12/2020

Resumen: Introducción. El cuidado de enfermería tiene una connotación ética reflejada en los atributos que implica el ejercicio del deber, establecido principalmente en el Código de ética profesional, mientras la ética de la virtud apela al desarrollo de valores. Autoras como Gilligan, Noddigns y Tronto han aportado desde la ética del cuidado, como referentes ético-teóricos al ámbito de una enfermería feminizada. Objetivo. Describir la contribución de la Ética del cuidado a la enfermería profesional. Metodología. Reflexión de tipo teórica que pretende aumentar la comprensión del cuidado a partir de los marcos conceptuales y epistemológicos desarrollado por las autoras revisadas. Resultados y discusión. El manuscrito se organizó describiendo: a) los conceptos considerados clave en la Ética del cuidado, b) Gilligan y la Ética del cuidado, y finalmente, b) el aporte realizado por Tronto y Noddigns que han ampliado la discusión sobre el cuidado a nivel universal, aportando al cuidado informal como profesional. Conclusiones. La Ética del cuidado ha permitido desarrollar marcos conceptuales que facilitan la comprensión del cuidado de manera universal, dándole un estatus fundamental para la vida en sociedad. Recuperar las virtudes éticas para el cuidado en un justo equilibrio con el deber, contribuye a que la enfermería revalore lo emocional en la relación de ayuda que establece con los pacientes y las comunidades.

Palabras claves: ética, ética de enfermería, empatía, cuidados de enfermería, Desarrollo moral. 


\begin{abstract}
Introduction. Nursing care has an ethical connotation reflected in the attributes that the exercise of duty implies, established mainly in the Code of Professional Ethics, while the ethics of virtues appeals to the development of values. Authors such as Gilligan, Noddigns and Tronto have contributed from the ethics of care, as ethical-theoretical references to the field of feminized nursing. Objective. Describe the contribution of the Ethics of care to professional nursing. Methodology. A theoretical reflection that aims to increase the understanding of care based on the conceptual and epistemological frameworks developed by the authors reviewed. Results and Discussion. The manuscript was organized describing the a) concepts considered key in the Ethics of care, b) Gilligan and the Ethics of care, and finally, b) the contribution made by Tronto and Noddigns who have broadened the discussion about care at a universal level, contributing to informal care as a professional. Conclusions. The Ethics of Care has allowed the development of conceptual frameworks that facilitate the understanding of care in a universal way, giving it a fundamental status for life in society. Recovering the ethical virtues for care in a fair balance with duty, contributes to nursing re-evaluating the emotional in the helping relationship it establishes with patients and communities.
\end{abstract}

Keywords: ethics, nursing, empathy, nursing care, moral development.

Resumo: Introdução. O cuidado de enfermagem tem uma conotação ética que se reflete nos atributos que o exercício do dever implica, estabelecidos principalmente no Código de Ética Profissional, enquanto a ética das virtudes apela ao desenvolvimento de valores. Autores como Gilligan, Noddigns e Tronto contribuíram a partir da ética do cuidado, como referenciais éticoteóricos para o campo da enfermagem feminizada. Objetivo. Descrever a contribuição da Ética do Cuidado para o profissional de enfermagem. Metodologia. Reflexão de tipo teórico que visa ampliar a compreensão do cuidado a partir dos referenciais conceituais e epistemológicos desenvolvidos pelos autores revisados. Resultados e discussão. O manuscrito foi organizado descrevendo a) conceitos considerados fundamentais na Ética do cuidado, b) Gilligan e a Ética do cuidado, e por fim, b) a contribuição de Tronto e Noddigns que ampliaram a discussão sobre o cuidado em nível universal, contribuindo para o cuidado informal como profissional. Conclusões. A Ética do Cuidado tem permitido o desenvolvimento de marcos conceituais que facilitam a compreensão do cuidado de forma universal, conferindo-lhe um estatuto fundamental para a vida em sociedade. Recuperar as virtudes éticas para o cuidado em justo equilíbrio com o dever, contribui para a enfermagem reavaliar o emocional na relação de ajuda que estabelece com o paciente e a comunidade.

Palavras-chave: ética, ética em enfermagem, empatia, cuidado de enfermagem, desenvolvimento moral.

Correspondencia: Edith Rivas Riveros, e-mail: edith.rivas@ufrontera.cl. 


\section{Introducción}

La profesión de enfermería lleva implícito el concepto de cuidado, tema vital para la subsistencia de la humanidad y las relaciones solidarias entre las personas. Desde esta perspectiva, cuidar es una actividad indispensable para la humanidad, toda vez que no se trata solo de supervivencia, sino de promover y desarrollar todas aquellas actividades que velan por el bien común de las personas y de los grupos. Según Nightingale, toda mujer en algún momento de su vida ejercerá como enfermera de una u otra forma, ya que la enfermería consiste en acompañar o asumir responsablemente los cuidados de otra persona (1). Así el cuidado de enfermería se define desde una visión holística de la humanidad en que la persona es un todo conformado por distintas dimensiones del ser; física, psicológica, social y espiritual. Jasemi et al., (2) al respecto resalta que es posible encontrar enfermeras con gran capacidad para mantener un cuidado equilibrado entre las dimensiones humanas, mientras otras tienen un mayor interés por el cuidado físico, en que aspectos de la relación del cuidado podrían debilitarse. Por lo que los aspectos éticos deben ser una preocupación permanente considerando lo relevante de tomar decisiones en favor y beneficio de los pacientes. Feito por su parte, plantea que la tarea profesional de las enfermeras es una práctica moral en la que el cuidado cobra su máxima vigencia, dado que justifica y legitima el valor social que aporta la profesión a la sociedad a través de los cuidados (3).

La moralidad dice relación con estándares de conducta que distinguen lo correcto de lo incorrecto, a diferencia de la ética que tiene por tarea pensar en el carácter ideal de la conducta humana. La importancia de este punto es que el carácter ético de una profesión permite que esta sea reconocida socialmente, por lo que la profesión de enfermería define el cuidado como su acción social esencial $(3,4)$.

La mayor importancia dada en las últimas décadas a la ética, la establece como eje central de la profesión de enfermería, así como también cobra importancia en otras profesiones de la salud (3). Contar, por tanto, con una buena formación y conocimiento de la ética profesional contribuye a mejorar las prácticas de cuidado poniendo en el centro del quehacer al paciente, persona y comunidades. Mientras que el mayor empoderamiento de los pacientes sobre sus derechos presiona al sistema de cuidados para que las enfermeras sean más empáticas, sensibles a su dolor y a sus necesidades (5). Reflexionar sobre la práctica clínica, contribuye en este sentido a que las enfermeras se detengan a mirar con más atención el ¿cómo nos estamos relacionando con el paciente? y ¿cómo hacemos una eficiente gestión de recursos sin que afecte la humanización de los cuidados? Aspectos como escuchar al paciente, su singularidad a procesos de cambio por enfermedad, su respuesta emocional, psicológica e intelectual, son tan importantes como identificar y comprender cambios fisiopatológicos, para establecer los objetivos del cuidado e identificar las actuaciones apropiadas a desarrollar, lo que ayuda a focalizar este esfuerzo (5).

En efecto, priorizar la racionalidad y la gestión pensada solo desde la práctica profesional, sin la debida consideración de los aspectos psicológicos emocionales, podría provocar dificultades para tomar decisiones que beneficien verdaderamente al paciente. Por lo que reconocer la importancia de lo emocional en la gestión de los cuidados es uno de los aspectos claves para avanzar en un cuidado humanizado. Eludir a la "Ética del cuidado", permite según las autoras reflexionar sobre los fines y medios de las tareas asistenciales, enfatizando en los valores que se pretende salvaguardar (3). Esto debiese ser una reflexión habitual que viene a compensar la preeminencia de las propuestas que se han impuesto mayormente desde la modernidad. En esos 
modelos, denominados "éticas de la justicia" la virtud queda restringida al ámbito privado referida a las creencias u opciones personales. Así el modelo de la justicia distributiva bajo el discurso de defensa de las ideas de libertad e igualdad que están en la base de los sistemas democráticos del estado de derecho deja en segundo plano la dimensión emocional y social. Estos últimos elementos componen la vida moral de las personas, pero que no tienen ubicación en un modelo de fundamentación racional en el que se pretende la universalización imparcial. En este contexto, la Ética del cuidado recupera estas dimensiones, reivindicando la actitud de comprensión y la preocupación por las personas (3).

Esto claramente, trasciende el ámbito disciplinar y se extiende a todas las profesiones, sin embargo, corresponde a las enfermeras estar permanentemente con el paciente, interpretar sus emociones y voluntad a través de la relación de ayuda. Así, le entrega elementos únicos para abogar por este y asegurarse que sus necesidades estén cubiertas de forma eficiente y de manera humanizada. Por lo tanto, se puede plantear que la "Ética del cuidado" ha servido de guía para actuar en un mundo con valores como la solidaridad y la empatía los que prevalecen a la falta de cuidado $(6,7)$.

La "Ética del cuidado" ha favorecido a todas las profesiones sanitarias al promover la autorreflexión y desarrollar conciencia social a partir de principios universales humanistas, algo que se logra interiorizar a partir de la práctica profesional y la experiencia. Es así que los profesionales enfrentados a dilemas éticos logran identificar sus implicancias, se dan cuenta de que las posibles respuestas no son tan evidentes, ni fáciles de encontrar y, además, que no existen respuestas unívocas (3).

En el siguiente artículo, las autoras procuran aportar a la comprensión de la Ética del cuidado y su contribución a la enfermería profesional. Para ello, se revisarán: a) conceptos clave utilizados en la Ética del cuidado, b) Gilligan y la Ética del cuidado y, b) Tronto y Noddign que han ampliado la discusión sobre el cuidado a nivel universal. Se refuerza con ello, la importancia de la ética para la profesión en momentos en que se discute la importancia del cuidado respetando la dignidad de las personas, y cómo afecta a las sociedades y economía de los países. El abordaje metodológico es documental, basado en revisión de la literatura que permite contextualizar los aspectos teóricos de interés, para cumplir con el propósito señalado (8).

\section{Metodología}

Tipo documental teórica basada en revisión de literatura intencionada enfatizando en la perspectiva Ética del cuidado. Esto permitirá contextualizar y fundamentar los aportes a la profesión de enfermería y el cuidado, así como develar nuevas preguntas y respuestas al corpus teórico existente (8). 


\section{Resultados y discusión}

\section{Cuidado de Enfermería y Ética: conceptos relacionados}

La enfermería considera a la persona como un sistema integral, único en un contexto socio cultural en que nace y que debe ser considerado y respetado en el quehacer de la acción profesional. Las determinantes sociales que incluyen las condiciones de vida de la población requieren necesariamente de un enfoque integral y sistémico, pero a la vez a partir de la perspectiva teórica de la profesión $(3,9)$. En este aspecto, las enfermeras deben estar preparadas para identificar y respetar pautas culturales, espirituales, sociales, demográficas y psicológicas de cada población, relevando formas y condiciones de vida de las personas, sus necesidades y de quienes los cuidan, valorando la diversidad humana. Es por ello, que las enfermeras han de nutrirse de saberes y actitudes que permitan que sus acciones de cuidado tengan pertinencia socio-cultural, sin exclusión de ningún tipo y respetando los derechos humanos de toda condición humana (9).

El concepto de cuidado desde la disciplina enfermera es base de sustentación de la discusión. Para Colliére, el cuidado de los pacientes representa una serie de actos que tienen por finalidad y función mantener a los seres humanos vivos y sanos para reproducirse y perpetuarse. Así, el cuidado consiste en mantener la vida asegurando la satisfacción de un conjunto de necesidades para el individuo, familia, y comunidad, que en continua interacción con su entorno vive experiencias de salud (10). El cuidado requiere de conocimientos que lo singularizan como cuidado humano y profesional, siendo sobre el que se articula la profesión de enfermería (11). Nigthingale fue capaz de darse cuenta de que el cuidado natural de las personas, basado en amor y dedicación por otros, no bastaban para fomentar la salud o curar una enfermedad, sino que el cuidado dependía de ciertas habilidades, experiencias y conocimientos específicos, sentando las bases para el cuidado profesional (12). Los estereotipos femeninos como fuente de cuidados naturales, también se asociaron a sumisión y subordinación, atributos descritos como propios de las mujeres. Esto es traspasado a la profesión de enfermería desde sus inicios como auxiliar del médico, con vocación de servir al enfermo, al médico y a la institución (12). De tal manera, esto trajo grandes efectos en su desarrollo futuro, pues el estatus profesional históricamente ha sido moldeado por relaciones de género que han limitado a las enfermeras para acceder a cargos de poder, con condiciones de trabajo y salarios que la mayoría de las veces son menores que los hombres (13).

El valor social de los cuidados realizado por mujeres, basado en experiencia vivida con su propio cuerpo, ejercido por madres, hermanas, abuelas o suegras y estrechamente relacionado con la reproducción y la maternidad, fueron marcando la forma de ejercer el cuidado natural, que por extensión alcanza a las enfermeras profesionales, para que sigan siendo abnegadas, generosas y desinteresadas, propio de una profesión mayoritariamente femenina (12).

Para la enfermería, el cuidado se considera la esencia de la disciplina que implica no solamente al sujeto de cuidado, sino a las enfermeras en una relación caracterizada por la bidireccionalidad (14). Kérouack plantea en este sentido, que "la práctica enfermera se centra en el cuidado de la persona y familia en continua interacción con el entorno" (15). En este escenario las enfermeras identifican junto a la persona cuidada las repercusiones del cuidado en su proceso salud-enfermedad e implementan estrategias en común acuerdo, con el objetivo de lograr la adaptación a una situación particular y la consecución del máximo nivel posible de salud. Cuidar es trabajar para atender a las personas, contando y partiendo de la propia persona $(11,16)$. Cuidar 
es acompañar a la persona en sus experiencias de vida y reconocer su dignidad por sobre técnicas y medios terapéuticos; es romper la dicotomía entre técnica y humanismo, ya que ambos aspectos deben coexistir de forma integrada (17).

Henderson esboza que el cuidado enfermero implica ofrecer asistencia en todas aquellas actividades que una persona no puede realizar por sí mismo, con el objetivo de contribuir a su salud y ayudarle a aumentar sus capacidades para llegar a un nivel de independencia en su vida cotidiana y adaptarse a su situación de enfermedad (1). La dedicación en el cuidar a un ser humano implica valores universales como la ternura, flexibilidad y generosidad (18). También, Feito destaca que la enfermería es una práctica moral, puesto que incorpora una actitud o comportamiento ético basado en una virtud moral reflejada en la solidaridad y preocupación por el bien de los seres humanos, y por la que se exige responsabilidad para dar mayor dignidad a las personas (19). Esta responsabilidad constituye un elemento intrínseco del cuidado a través del cual se ejercen los derechos humanos a la salud y la vida (3).

Los primeros códigos éticos han constituido la base ética de la práctica médica e influenciado otras disciplinas de las ciencias de la salud, tal como es el caso de enfermería. Si bien se constata una ética vinculada al cuidar humano, el nacimiento de la ética enfermera se sitúa en el mismo punto que el de la enfermería como profesión. Además del compromiso explícito de Nightingale y de su obra, las bases de la ética enfermera se encuentran ligadas a algunas corrientes que han emergido a lo largo de la historia. Las más influyentes han sido la ética prescriptiva, ética de la virtud y Ética del cuidado que constituyen referentes para la ética enfermera (20).

La ética prescriptiva o normativa es una corriente ética que apela a la naturaleza de la acción moral, utiliza normas generales para establecer que una acción es moralmente buena cuando está acorde a un principio universal, es decir, válida para todos los seres humanos y situaciones posibles. Será, por tanto, moralmente mala cuando contradice estas normas universales (21). Por otra parte, la ética de la virtud reúne una serie de discursos de la filosofía moral contemporánea que ha tratado de recuperar la categoría de virtud y que al mismo tiempo, busca estructurar un modelo para la comprensión y orientación de la moralidad, mientras la Ética del cuidado apela a los apegos y la responsabilidad (22).

En síntesis, no es posible lograr la excelencia clínica mejorando solo la calidad técnica de los cuidados en cuanto al uso de recursos y prestaciones, ya que se requiere también, de un desarrollo ético adecuado que defina el carácter de la profesión a través de la posibilidad de brindar al paciente acompañamiento, empatía, confidencialidad, es decir, los atributos que definen una buena relación terapéutica y de ayuda. Precisamente, si se logra dotar a los profesionales de enfermería de conocimiento técnico y humanista en un justo y sano equilibrio, se alcanzará la excelencia del cuidado profesional, la satisfacción de realización personal y colectiva (23).

\section{Origen de la Ética del Cuidado}

La Ética del cuidado tiene sus orígenes en la discusión de Gilligan con las investigaciones de Kohlberg, quien en el año 1978 llevó a cabo una investigación sobre el desarrollo moral para comprobar si al igual que en el desarrollo cognitivo de Piaget, existía un desarrollo moral secuencial y por etapas que proporcionara como resultado la maduración moral. Dicho modelo fue objeto de numerosas críticas en relación a la metodología utilizada. Quizás una de las detracciones más reveladoras fue la existencia de sesgo de género, ya que el psicólogo centró su estudio en los 
razonamientos de participantes hombres. Siendo esto, lo que impulsó a Gilligan, discípula de Kohlberg, a investigar la temática. Hasta entonces no existían investigaciones relacionadas con el desarrollo moral que incluyeran a las mujeres lo que para Gilligan era una omisión de verdades y una limitación en el desarrollo del concepto de "condición humana" (23).

El objetivo de la investigadora no era otro que "ensanchar la comprensión del desarrollo humano, valiéndose del grupo que se omitió en la construcción de la teoría, para llamar la atención hacia lo que falta en su versión" y, además, proporcionar "una base sobre la cual generar una nueva teoría que, potencialmente, pueda otorgar una visión más general de las vidas de ambos sexos" (23). Así en 1982 Gilligan publica "In a different voice: Psycological Theory and women 's Development", traducido como "La moral y la teoría psicología del desarrollo femenino", texto donde desafía la concepción tradicional sobre el desarrollo moral, poniendo en entredicho la presunta universalidad del paradigma evolutivo de Kohlberg, a la luz de las experiencias de las mujeres; hasta ese momento excluidas de los análisis teóricos sobre el desarrollo y la capacidad moral (24). Gilligan señaló que tanto los teóricos morales como los de la psicología habían "adoptado implícitamente la vida del hombre como norma, tratando de crear mujeres a base de un patrón masculino". De este modo, puso de manifiesto la necesaria incorporación de la experiencia femenina en la teoría moral y política, históricamente asociada a los hombres y al ámbito público con una orientación moral focalizada en la justicia y los derechos.

Desde esta perspectiva, Gilligan intenta expandir el ámbito de la moralidad proponiendo la noción de Ética del cuidado y sentando las bases para su posterior diálogo y complementación con la ética de la justicia (25).

Gilligan observó que las mujeres obtenían puntuaciones bajas y no solían llegar al nivel moral final. En un primer momento, dicha evidencia fue interpretada por Kohlberg como una incapacidad de la mujer para emitir juicios morales superiores, provocada por su emplazamiento en la sociedad, referido al espacio privado y doméstico, donde se desarrollan los temas del cuidado, afecto y responsabilidad.

Gilligan se adentró en la problemática a partir de dos premisas básicas de la teorización feminista: la realidad social se organiza en base a un sistema sexo-género que define una forma de percibir el mundo, y dicho sistema se basa en la dominación patriarcal en el que los hombres detentan poder sobre las mujeres. Preguntándose el porqué de este hecho, y no dando por sentado que se debía a una supuesta "inmadurez moral” de las mujeres, Gilligan mostró que en los estudios de Kohlberg no se consideraban las estructuras sociales de exclusión sexo-género, ni el hecho de que las experiencias concretas de los sujetos condicionan los modos que tienen de desarrollar sus razonamientos morales $(25)$.

En esta línea, se pone en entredicho la justicia universalista contemporánea basada en un modelo de ciudadanía construido a través de lo común y dejando de lado las diferencias, priorizando una moralidad sobre otra y a un grupo humano sobre el otro: hombres por sobre las mujeres, esfera pública sobre esfera privada, justicia y deber sobre cuidado y responsabilidad. Gilligan demostró que no existe un modo único de entender la moralidad, sino que "los caminos divergentes de desarrollo moral que siguen hombres y mujeres en términos de diferencias de experiencia" marcan diferentes modos de desarrollar la moral (25). Este aspecto es especialmente importante para la profesión, desde la mayor presencia de mujeres que formalmente se dedican al cuidado que hombres. 
La autora plantea que el concepto central de la Ética del cuidado es la responsabilidad y el actuar moral de las mujeres, que se centraría más que en juicios generales abstractos, en la responsabilidad que surge de la conciencia de formar parte de una red de relaciones de interdependencia. Esto debido a que su identidad está fuertemente constituida de manera relacional. Además, observó que existe una asociación entre el modo de razonamiento moral y la concepción del yo de hombres y mujeres. Estas últimas, cuando se describen a sí mismas lo hacen en términos de relación y no mencionan sus distinciones académicas o profesionales, al contrario de los hombres $(6,24)$. A partir de esta distinción, Gilligan elabora un cuadro del desarrollo moral en el ámbito de la Ética del cuidado. En el primer estadio, la mujer se concentra en el cuidado de sí misma. En el segundo estadio, el bien se identifica con el cuidado de los otros, período en la que se confunden el cuidado y el sacrificio. La tercera etapa comprende la transición de la femineidad a la adultez y consiste en aprender a cuidar de los demás como de sí misma, y asumir la responsabilidad por sus opciones (6).

El aporte a la profesión de enfermería a partir de lo planteado por Gilligan se basa en la responsabilidad como concepto central de la ética del cuidado, éste es el eje que guía el actuar de la profesión de enfermería, entendiendo dicha responsabilidad como una acción en forma de ayuda, $(6,26)$ y no solo como una cuestión de deber y justicia (3). Tiene que ver con situaciones reales, verdaderas como las necesidades de las personas, el deseo de evitar el daño, la circunstancia de ser responsable de otro, tener que proteger, atender a alguien. La moralidad como compromiso deriva precisamente de la certeza de que el bienestar e incluso la supervivencia requieren algo más que justicia. En este aspecto, la teoría de Gilligan resalta el papel moral de las emociones, afectividad y todas las esferas de la personalidad $(27,28)$. Es una teoría, o un conjunto de reflexiones morales que se centra en el cuidado como imperativo moral. Le concede un lugar preponderante al bien de las personas, personas con la que se entabla una relación, relación que respeta las emociones humanas (29). Enfatizando en las necesidades de las personas en situación de vulnerabilidad y dependencia, dando prioridad a su cuidado (30,31). El modo de responder a las necesidades de la persona, por tanto, se basa en analizar las emociones y las relaciones interpersonales vinculadas (30).

\section{Ética del Cuidado: mirada de otros autores}

A partir de la propuesta teórica de Gilligan se han producido importantes debates teóricos, en que filósofos(as) y sociólogos(as) han enfatizado en la importancia de lo relacional en el cuidado. En este aspecto, Camps menciona que más que el deber, lo importante en el cuidado es la relación con las personas y su aplicación situacional, mezclando la racionalidad con la emotividad para centrarse en el compromiso directo y personal con los otros (32). A su vez, Lévinas refuerza lo central de lo emocional en el cuidado, en donde la sensibilidad es lo que sustenta la responsabilidad y la vigilancia extrema (33). Contrariamente, otros(as) autores han criticado abiertamente la Ética del cuidado haciendo mención de que se trata de una teoría subdesarrollada (34), que es una simplificación que adolece de falta de adhesión a deberes y al principio de justicia (35), y que es "femenina" sosteniendo que la ética es universal y no pertenece a grupo alguno (34).

Para la profesión de enfermería la Ética del cuidado descubre aspectos importantes del desarrollo moral de la mujer aplicables a la profesión, dado que la profesión está conformada principalmente por mujeres (36). Noddings, conocida por su trabajo en la filosofía de la educación, 
ha planteado la importancia del cuidado relacional como un aspecto fundamental y vital para la vida en sociedad (36). Su trabajo está anclado en un análisis del cuidado y su lugar en la ética. Su argumento parte de la idea de que el cuidado es básico en la vida humana, y que todas las personas desean ser cuidadas. Sin embargo, el cuidado exige un esfuerzo de ampliación para motivar e incentivar el cuidado en las relaciones sociales. Para la autora, la Ética del cuidado tiene sus raíces prácticas en la historia y en las tradiciones de las mujeres; no es defender el feminismo (36). Al respecto menciona: "No tengo ninguna idea de si las mujeres son por naturaleza, más afectivas que los hombres. Yo lo dudo, pero creo que se puede aprender estudiando la tradición de cuidado que ha sido en gran parte la historia de las mujeres. El hecho de que el cuidado hubiese surgido de las condiciones de subordinación no es razón para rechazarlo, sino para evaluarlo y acariciar sus mejores rasgos, y para animar a los jóvenes a que entren en su espíritu y práctica" (38). Noddings plantea que la ética tiene sus raíces en los sentimientos, aunque no rechaza la razón como guía para la vida moral (38). "Lo que nos motiva no es la razón, sino el sentimiento hacia el otro" (39). Este sentimiento hacia el otro o empatía, que se hace patente en la relación entre cuidador y persona cuidada, conlleva apreciar las cosas desde su punto de vista y no actuar en base a normas o principios del cuidador (30). Sostiene, además, que la moralidad es una virtud activa que requiere de por lo menos dos sentimientos: el primero corresponde al sentimiento de cuidado natural innato en los seres humanos, que posibilita que actuemos en nombre de otras personas porque queremos hacerlo. El cuidado de una madre y los esfuerzos en nombre de su hijo no suelen considerarse éticos sino naturales; el segundo corresponde al sentimiento de cuidar ético y se fundamenta en el cuidar natural, que surge como respuesta al recuerdo del cuidar natural cuando coexiste un conflicto entre los deseos propios y el actuar, de acuerdo al cuidado de los otros (38). Para desarrollar estos conceptos, la autora apela a la distinción entre actuar por deseo y por deber. Cuando una persona abraza a un amigo porque necesita un abrazo en un momento determinado se actúa "por deseo", esto es guiado por el cuidado natural. Cuando abraza a una persona pese a que desearía no hacerlo actúa por deber, es decir, en base al cuidar ético.

El cuidar natural y/o el cuidar ético también son explicados por el tipo de relación que se desarrolle con la persona cuidada (30). Ahora bien, para la autora existen diversos niveles de relacionalidad pues las personas se encuentran rodeadas de círculos concéntricos de relaciones afectivas y sociales. El primero es el círculo íntimo el que mayor influencia tiene sobre las personas y que está compuesto por los seres que amamos, en donde el cuidar natural se hace más evidente. No obstante, incluso en estos contextos puede presentarse el caso de requerir del cuidar ético. Otro círculo, es el que está constituido por personas a las que tenemos consideración y estima, pero con quien no mantenemos una relación estrecha (30). La ayuda se expresaría en relación con cómo se siente en ese momento, aquello que la otra persona espera de nosotros y qué tipo de relación se tenga con ésta. Por último, existen otros tipos de relaciones que Noddings denomina "extraño próximo" y "extraño remoto". En ambos casos se trata de personas que no se conocen personalmente, pero que pueden llegar a ser conocidos si algo los une de algún modo con ellos(as), por ejemplo; vecinos, compañeros de clase, etc. Mientras que extraño remoto es aquel con el que nunca se tendrá ningún tipo de relación, pero que de igual forma nace la necesidad de cuidar (30).

El diálogo es para Noddings el que permite relacionarse con otros y conocer sus necesidades y deseos. El cuidador ha de prestar atención a la persona que ha de cuidar para entender cómo y por qué desea ser cuidada de una determinada manera, y se ha de dedicar por completo a esta tarea. Por otro lado, la persona cuidada debe apreciar estos esfuerzos que realiza 
el cuidador por comprenderla y cuidarla de modo que ha de intentar informarle minuciosamente de qué forma y por qué ha de hacerlo de un modo concreto en relación con sus propias circunstancias (30). Noddings ha desarrollado un pensamiento que es útil para construir una teoría libre de sesgos de género, sus planteamientos ponen de manifiesto la necesidad de transformar las pautas socioculturales a favor de la Ética del cuidado. El desarrollo humano necesita sobre todo del cuidado que se recibe de las personas con las que se está vinculada afectivamente (40). Es ella, quien con su propuesta conduce el cuidado desde una posición femenina específica hacia la educación moral en su totalidad. Femenina, porque como ya se ha mencionado, el cuidado históricamente ha sido parte principalmente de la vida de las mujeres, como madres y esposas a cargo del hogar. Pero ella aclara que no se trata de un rasgo esencial de constitución de la mujer, sino que es una experiencia abierta a todo ser humano, especialmente a aquellos que están en posición de cuidar a otros. La ética del cuidado no desconoce a los agentes morales masculinos, considerándolo el ideal para todos los agentes morales (37). Noddings define el cuidado natural como: "la condición humana que nosotros, consciente o inconscientemente, percibimos como buena" (38). "Es esa condición la que nosotros anhelamos y por la que nos esforzamos, y es nuestro anhelo por estar en esa relación especial el que mantiene la motivación de ser moralmente buenos" (37).

Otra destacada autora que ha investigado la ética del cuidado es Joan Tronto que ha explorado las intersecciones de la ética del cuidado, la teoría feminista y la ciencia política. Ella sanciona una ética de cuidado feminista para frustrar la acumulación de poder a los poderosos existentes, y para aumentar el valor de las actividades que legitiman el poder compartido. Identifica los límites morales que han servido para privatizar las implicaciones de la ética del cuidado, y destaca las dinámicas políticas de las relaciones de cuidado que describen, por ejemplo, la tendencia de las mujeres y otras minorías a realizar el trabajo de cuidado de manera que beneficie a la elite social. Tronto acuña la frase "irresponsabilidad privilegiada", para describir el fenómeno que permite a los más aventajados de la sociedad comprar servicios de cuidado, delegar el trabajo de cuidado y evitar la responsabilidad por la idoneidad de la atención práctica (41). La autora entiende el cuidado como una "actividad genérica que comprende todo lo que hacemos para mantener, perpetuar, reparar nuestro mundo de manera que podamos vivir en él lo mejor posible. Este mundo comprende nuestro cuerpo, nosotros mismos, nuestro entorno y los elementos que buscamos enlazar en una red compleja de apoyo a la vida". A partir de esta forma de aplicar la ética del cuidado, Fisher y Tronto, plantean cuatro fases funcionales del cuidado $(3,26)$ :

a) Caring about: implica el reconocimiento de las necesidades, el requisito moral para dicho interés se describió como "atención",

b) Caring for: aceptar la responsabilidad supone asumirla en relación con la necesidad identificada, así como determinar la naturaleza de la respuesta a aportar.

c) Care giving: es la tarea real del cuidado, con la competencia como la noción moral necesaria para cubrir dichas necesidades.

d) Care receiving: recibir el cuidado, con la cualidad moral de la capacidad de respuesta.

Posteriormente añade una quinta fase, el "caring with" (cuidar con) en esta fase se hace referencia al hecho de que mientras se produce el cuidado, las personas tienden a confiar en la 
provisión continuada de dicho cuidado. Sus cualidades morales son la confianza y la solidaridad definida culturalmente. Considera el cuidado activo caracterizando una actividad singular o un proceso. Es a la vez una práctica y una disposición $(3,26)$. De ese modo, Tronto afirma que el ámbito del cuidado es inmenso y reconoce la dificultad que existe para delimitar su campo, que se materializa en actividades concretas, orientadas al cuidado y autocuidado. En el cuidado es decisivo tener en cuenta las necesidades del otro para guiar la acción, implica una forma de relación activa $(3,26)$.

En este punto son muchos los(as) autores(as) que han destacado las bondades de la ética del cuidado reconociendo que, a partir de ésta, ha existido un cambio a favor del cuidado formal e informal, en donde confluyen virtudes como la solidaridad, atención, responsabilidad, competencia, capacidad de respuesta y confianza. La atención de cuidar, por tanto, requiere escuchar y comprender las circunstancias de aquellos que buscan ayuda. La responsabilidad incluye asumir la acción en base a la atención y particularidades de las necesidades de los pacientes y de las personas cuidadoras, dedicando tiempo a construir confianza (42). Tronto destaca especialmente este aspecto al referirse a la naturaleza de la relación entre quien provee el cuidado y quien es sujeto del mismo, como proceso interactivo (26).

Ampliar la capacidad de comprensión moral permite el acercamiento al otro, cultivando actitudes de relación, habilidades de comunicación y resolución de problemas, considerando la singularidad y particularidad de cada caso, poniendo de relieve la autenticidad de la persona sin olvidar lo universal y objetivo (20). Aplicado al cuidado formal, la importancia de la relación entre enfermera y paciente potencia el contacto haciéndolo más humano, fundamentada en la responsabilidad hacia el otro $y$, en consecuencia, en su atención integral mediante el fomento de actitudes adecuadas que dota a quien la practica de un compromiso profesional y moral (43).

De acuerdo con lo expuesto, la ética del cuidado implica que existe un significado moral en los elementos fundamentales de las relaciones y las dependencias de la vida humana. Normativamente busca mantener relaciones al contextualizar y promover el bienestar de los cuidadores y receptores en una red de relaciones sociales. Lo más a menudo definido como una práctica o virtud en lugar de una teoría como tal, "atención" implica mantener el mundo y satisfacer las necesidades personales y de los demás (41). Se basa en la motivación para cuidar de aquellos que son dependientes y vulnerables, y se inspira tanto en los recuerdos de ser atendidos como en las idealizaciones del yo. Afirma, además, la importancia de la motivación, emoción y el cuerpo en la deliberación moral, así como el razonamiento a partir de los detalles (41).

\section{Conclusiones}

La ética el cuidado ha contribuido al fortalecimiento de la acción de cuidar, autoras como Gilligan, y posteriormente Tronto y Noddings han desarrollado marcos conceptuales que facilitan la comprensión del cuidado de manera universal, elevando su estatus como una acción fundamental para la vida en sociedad. Recuperar las virtudes éticas para el cuidado en un justo equilibrio con el deber, contribuye a que los profesionales de enfermería revaloren la relación de ayuda que se establece con los pacientes, dado que posee una formación integral que le entrega los conocimientos, preparación, motivación y sensibilidad moral para cuidar. 
La ética del cuidado está, por lo tanto, íntimamente relacionada con la profesión de enfermería responsable del cuidado formal de las personas y las comunidades, como profesión históricamente conformada por mujeres, con gran sensibilidad emocional y moral natural, e injustamente cargando con los prejuicios asociados a su género, desvalorizando e invisibilizando el cuidado característico de un mundo que resalta por sobre todo la productividad. Se tiene por ello, la necesidad de reflexionar sobre el espacio que se le ha dado a lo emocional en el cuidado formal, como un rasgo íntimo y exclusivo del carácter social de la profesión.

Las voces que se han alzado a nivel global, como Gilligan, Noddings y Tronto, han contribuido sentando las bases teóricas para poner el tema en debate de forma transversal, dando al cuidado un estatus que lentamente comienza a llamar la atención de los responsables de las políticas públicas en los países. Dado que el cuidar es una tarea esencial para el bien común de la sociedad, para la mantención de principios cívicos como la solidaridad, y por tanto el considerarla en las políticas públicas afecta favorablemente la economía de los países. El cuidar no es una carga para las personas y el Estado, sino el reflejo de una sociedad que se desarrolla de manera solidaria y con respeto por los derechos humanos fundamentales de todas las personas sin exclusión.

\section{Referencias Bibliográficas}

1. Raile M. Modelos y Teorías en Enfermería. 8a ed. Madrid España: Elseiver; 2015.

2. Jasemi M, Valizadeh L, Zamanzadeh V, Keogh B. A Concept Analysis of Holistic Care by Hybrid Model. Indian journal of palliative care[internet]. 2017 [citado el 25. Oct. de 2020]; 23(1): 71-80. Disponible desde: https://doi.org/10.4103/0973-1075.197960

3. Feito LG. Ética del cuidado en las profesiones sanitarias. Revista de estudios sociales y de sociología aplicada [internet]. 2017.[citado el 25 de Oct. de 2020];187(0): 29-47. Disponible en: https://caritas-web.s3.amazonaws.com/main-files/uploads/2019/01/Doc-Social-187.pdf

4. Ortiz GM. Sobre la distinción entre ética y moral. Isonomía [Internet]. 2016 [citada el 05 de octubre de 2019]; (45), 113-139. Disponible en: http://www.scielo.org.mx/scielo.php?script=sci_arttext\&pid=S140502182016000200113\&ln $\mathrm{g}=\mathrm{es} \& \mathrm{t} \operatorname{lng}=\mathrm{es}$

5. Blasco ML, Ortiz LR. Ética y Valores de Enfermería. Rev Enferm Inst Mex Seguro Soc. [Internet]. 2016 [citado el 05 de Oct. de 2019]; 24(2):145-9. Disponible en: http://revistaenfermeria.imss.gob.mx/editorial/index.php/revista_enfermeria/article/viewFile/ $104 / 152$

6. Gilligan C. In a Different Voice: Psychological Theory and Women's Development. Thirtyeighth printing. United State: Harvard University Press; 2003.

7. García LM. La ética del cuidado y su aplicación en la profesión enfermera. Acta bioeth. [Internet]. 2015 Nov [citado el 29 de Oct. de 2020]; 21( 2 ): 311-317. Disponible en: http://dx.doi.org/10.4067/S1726-569X2015000200017

8. Guirao SG. Utilidad y tipos de revisión de literatura. Ene [Internet]. 2015 [citado 20 de Oct. de 2020] ; 9(2). Disponible en: http://dx.doi.org/10.4321/S1988-348X2015000200002

9. Vitón de Antonio M, Castro de la Iglesia F. Contextos socioculturales del cuidar y ejercicio ético. Reflexividad y compromisos pedagógicos para la democratización de vida compartida Sociocultural contexts of caring and ethical exercise. Saber \& Educar [internet]. 2019 [citado el 28 de oct. de 2020]; O(27). Disponible en: doi:http://dx.doi.org/10.17346/se.vol0.361 
10. Collière M. Promover la vida. México: Interamericana McGraw-Hill; 2003.

11. Bratz J, Sandoval MR. Competencias éticas para el desarrollo de cuidados en enfermería. Rev Bras Enferm [Internet]. 2018 [citado 27 de Oct. de 2020];71(suppl 4):1915-20 . Disponible en: https://www.scielo.br/pdf/reben/v71s4/es_0034-7167-reben-71-s4-1810.pdf

12. Zúñiga Y, Paravic T. El género en el desarrollo de la enfermería. Rev Cubana Enfermer [Internet]. 2009 [citado el 1 de jul. de 2019]; 25(1-2). Disponible en: http://scielo.sld.cu/scielo.php?script=sci_arttext\&pid=S0864-03192009000100009\&lng=es.

13. Clayton KH, Griffiths H, Schutz S, Humbert A, McIlroy R. Gender and Nursing as a Profession Valuing nurses and paying them their worth[internet]. Reino Unido: Royal Colege of Nursing, Oxford Brookes University; 2020 [citado el 24 de oct. de 2020]. Disponibl en: https://www.rcn.org.uk/professional-development/publications/pub-007954

14. Baéz FH, Nava VN, Ramos LC, Medina OL. El significado de cuidado en la práctica profesional de enfermería. Aquichan [Internet]. 2009 [citada el 10 de julio de 2019]; 9(2):127134. Disponible

en: http://aquichan.unisabana.edu.co/index.php/aquichan/article/view/1476/1676

15. Kérouack S, Pepin J, Ducharme F, Duquette A, Major F. El pensamiento enfermero. $1^{\circ}$ ed. Barcelona España: Masson; 1996.

16. Busquets MS. La importancia ética del tenir cura. Annals de Medicina [Internet]. 2008 [citada el 10 de julio de 2019]; 91(2):71-74. Disponible en: https://core.ac.uk/download/pdf/78634475.pdf

17. Busquets MS. La autonomía en el cuidado de la salud. Revista de debat polític. Elements de Bioètica [Internet]. 2004 [citada el 10 de julio de 2019]; 9:115-127. Disponible en: http://www.fcampalans.cat/uploads/publicacions/pdf/9_9.pdf

18. Meza GS. El cuidado para la humanidad: una mirada desde la antropología de la ternura. Cultura de los cuidados[internet]. 2019[citado el 30 oct. de 2020]; 0(52): 153-166. Disponible en: https://doi.org/10.14198/cuid.2018.52.14

19. Feito LG. Los cuidados en la ética del siglo XXI. Enfermería Clínica [Internet]. 2005 [citada el 12 de julio de 2019]; 15(3):167-74. Disponible en: http://www.academia.edu/24972539/Los_cuidados_en_la_\%C3\%A9tica_del_siglo_XXI

20. García V, Paredes P, Rivas ER. Aproximaciones a la ética y responsabilidad profesional de enfermería. Rev. iberoam. Educ. investi. Enferm[internet]. 2012 [citado el 29 de oct. de 2020]; 2(4):48-57. Disponible

en: https://www.enfermeria21.com/revistas/aladefe/articulo/55/aproximaciones-a-la-etica-yresponsabilidad-profesional-de-enfermeria/

21. Beck H. ¿Ética normativa o ética de situación?. Invenio [Internet]. 2004 [citado el 12 de julio de 2019]; 7(12): 39-44. Disponible en: https://www.redalyc.org/pdf/877/87701204.pdf

22. Díaz AG. La ética de la virtud y la bioética. Revista Colombiana de Bioética [Internet]. 2009 [citado el 26 de junio de 2019]; 4(1):93-128. Disponible en: http://www.redalyc.org/pdf/1892/189214300005.pdf

23. García LM. La ética del cuidado y su aplicación en la profesión enfermera. Acta bioeth [Internet]. 2015 [citado el 20 de junio de 2019]; 21(2): 311-317. Disponible en: http://dx.doi.org/10.4067/S1726-569X2015000200017

24. Fascioli A. Ética del cuidado y ética de la justicia en la teoría moral de Carol Gilligan. Revista ACTIO [Internet]. 2010 [citada el 20 de junio de 2019]; 12: 41-59. Disponible en: http://www.actio.fhuce.edu.uy/images/Textos/12/Fascioli12.pdf 
25. Medina MV. La ética del cuidado y Carol Gilligan: una crítica a la teoría del desarrollo moral de Kohlberg para la definición de un nivel moral postconvencional contextualista. Daimon Revista Internacional de Filosofía [Internet]. 2016 [citada el 20 de junio de 2019]; 67: 83-98. Disponible en: https://doi.org/10.6018/199701

26. Domínguez C, Kohlen H, Tronto J. El futuro del cuidado. Comprensión de la ética del cuidado y práctica enfermera[internet]. $1^{\text {a }}$ ed. Barcelona: Ediciones San Juan de Dios-Campus Docent; 2018. Disponible en: https://pbcoib.blob.core.windows.net/coib-publish/invar/d23d413742f4-4331-924e-b660473acf64

27. Alvarado AG. La ética del cuidado. Aquichán [Internet]. 2004 [citada el 15 de julio de 2019]; 4(1):30-39. Disponible http://www.scielo.org.co/scielo.php?script=sci_arttext\&pid=S165759972004000100005\&lng=en

28. Honderich T, editor. Enciclopedia Oxford de filosofia. Esparta Madrid. Editorial Tecnos; 2001.

29. Vielba J. Ética y enfermería en: Ética de las profesiones: Enfermería. España, Bilbao. Editorial Descleé De Brouwer S.A; 2001.

30. Ramos SP. La ética del cuidado: valoración crítica y reformulación. Laguna Revista Filosofía [Internet]. 2011 [citada el 26 de junio de 2019]; 29:109-122. Disponible en: https://riull.ull.es/xmlui/bitstream/handle/915/12671/L\%20_29_\%282011\%29_07.pdf?seque nce $=1 \&$ isAllowed $=\mathrm{y}$

31. Pinedo IC, Yáñez JC. Las emociones y la vida moral: una lectura desde la teoría cognitivoevaluadora de Martha Nussbaum. Veritas[internet].2017[citado 28 de oct. de 2020]; (36):4772. https://dx.doi.org/10.4067/S0718-92732017000100003

32. Camps V. El siglo de las mujeres. Madrid: Ediciones Cátedra; 1998.

33. Idareta FG, Úriz MP. Aportaciones de la ética de la alteridad de E. Lévinas y la ética del cuidado de C. Gilligan a la intervención en trabajo social. Alternativas [Internet]. 2012 [citado el 20 de julio de 2019]; 19:33-44. Disponible en: https://rua.ua.es/dspace/bitstream/10045/26718/1/Alternativas_19_02.pdf

34. Jana AA. "Elementos fundantes de la ética del cuidado y su posible aporte para la relación clínica" [tesis en Internet] [Santiago]: Universidad De Chile; 2008 [consulta el 20 de julio de 2019]. Disponible en: http://repositorio.uchile.cl/bitstream/handle/2250/133716/Elementosfundantes-de-la-etica-del-cuidado-y-su-posible-aporte-para-la-relacion clinica.pdf?sequence $=1 \&$ isAllowed $=y$

35. Ferrer J, Álvarez J. Para fundamentar la Bioética. España. Bilbao. Editorial Descleé de Brouwer; 2003.

36. Rivera O. El cuidado: una alternativa en la educación moral [tesis en Internet] [Bogotá]: Pontificia Universidad Javeriana; 2008 [citada el 20 de mayo de 2019]. Disponible en: https://www.javeriana.edu.co/biblos/tesis/filosofia/tesis09.pdf

37. Noddings N. Educating moral people: a caring alternative to character education. Nueva York: Teachers College Press; 2002.

38. Noddings N. Caring: a feminine approach to ethics and moral education. Berkeley, California: University of California Press; 1984.

39. Noddings N. La educación moral. Propuesta alternativa para la educación del carácter. Buenos Aires, Argentina: Amorrortu; 2009.

40. Vázquez VV. La educación y la ética del cuidado en el pensamiento de Nel Noddings. [tesis en Internet] [Valencia]: Universidad de Valencia; 2009 [consulta el 20 de diciembre de 2019]. 
Disponible

en: https://www.researchgate.net/publication/50819403_La_educacion_y_la_etica_del_cuidado_ en_el_pensamiento_de_Nel_Noddings/download).

41. Sander MS. Care Ethics [Internet]. Internet Encyclopedia of Philosophy. Arizona State University. USA [consulta el 15 de julio de 2019]. Disponible en: https://www.iep.utm.edu/care-eth/

42. Barnes M, Brannelly T. Lograr atención y justicia social para personas con demencia. Ética de enfermería [Internet]. 2008 [consulta el 20 de enero de 2020]; 15 (3), 384-395. Disponible en: https://doi.org/10.1177/0969733007088363

43. Davis A. El cuidar y la ética del cuidar en el siglo XXI: qué sabemos y qué debemos cuestionar [Internet]. Col·legi Oficial d'Infermeria de Barcelona. Barcelona. 2011 [citada el 15 de julio de 2019]. Disponible en: https://pbcoib.blob.core.windows.net/coib-publish/invar/6b0331eba87d-4fa3-acc7-be341a1e709c

Publicación del Programa Magíster en Enfermería mención Gestión del Cuidado. Universidad de La Frontera.

Cómo citar: Yáñez Flores, K., Rivas Riveros, E., y Campillay Campillay, M. Ética del cuidado y cuidado de enfermería. Enfermería: Cuidados Humanizados. 2021; 10(1): 03-17. Doi: https://doi.org/10.22235/ech.v10i1.2124

Contribución de los autores: a) Concepción y diseño del trabajo, b) Adquisición de datos, c) Análisis e interpretación de datos, d) Redacción del manuscrito, e) Revisión crítica del manuscrito. K. Y. F. ha contribuido en a, b, c, d, e; E. R. R. en c, d, e y M. C. C. en c, d, e.

Editora científica responsable: Dra. Natalie Figueredo 